\title{
CONVEXITY PROPERTIES OF DISTINGUISHED EIGENVALUES OF CERTAIN CLASSES OF OPERATORS
}

\author{
B. NAJMAN
}

(Communicated by Palle E. T. Jorgensen)

\begin{abstract}
We prove two convexity results:
(1) Let $A(\varepsilon)=A_{0}+\varepsilon A_{1}$ be a family of selfadjoint operators in a Krein space with separated spectrum so that the maximum $\lambda_{-}(\varepsilon)$ of the spectrum of negative type of $A(\varepsilon)$ is an isolated simple eigenvalue. Then $\lambda_{-}(\varepsilon)$ is convex.

(2) Let $\lambda_{-}(\varepsilon)$ be the left distinguished eigenvalue of the generalized eigenvalue problem $\left(A_{0}+\varepsilon A_{1}\right) x=\lambda B x$ where $A_{1}$ and $B$ are real diagonal matrices and $A_{0}$ is an irreducible essentially nonnegative matrix. Then $\lambda_{-}(\varepsilon)$ is convex.

In both cases $\lambda_{-}(\varepsilon)$ is strictly convex unless it is linear.
\end{abstract}

\section{INTRODUCTION}

Let $A_{0}$ and $A_{1}$ be operators in a Banach space $X$ such that $A_{1}$ is $A_{0}$ bounded. Let $\lambda_{0}$ be an isolated simple eigenvalue of $A_{0}$; it is well known that for $|\varepsilon|$ small enough there exists a unique simple eigenvalue $\lambda(\varepsilon)$ of the operator $A(\varepsilon)=A_{0}+\varepsilon A_{1}$ with the property $\lim _{\varepsilon \rightarrow 0} \lambda(\varepsilon)=\lambda_{0}$. Moreover $\lambda(\varepsilon)$ is an analytic function of $\varepsilon$ in a neighborhood of $\varepsilon=0$. We are interested in its convexity properties.

The following special cases have been investigated:

(a) If $X$ is a Hilbert space, $A(\varepsilon)$ self-adjoint for $\varepsilon$ real and $\lambda_{0}$ is the maximum (the minimum, resp.) of the spectrum of $A_{0}$ then $\lambda(\varepsilon)$ is convex (concave, resp.). Moreover, it is strictly convex (strictly concave, resp.) unless $A_{0} x_{0}=\lambda_{0} x_{0}$ implies $A_{1} x_{0}=\lambda_{1} x_{0}$, hence $\lambda(\varepsilon)$ is linear.

(b) If $X=\mathbf{C}^{n}, A_{0}$ is an irreducible essentially nonnegative matrix (i.e. all its off-diagonal elements are nonnegative), $A_{1}$ is a real diagonal matrix and $\lambda_{0}$ is the principal eigenvalue of $A_{0}$ (i.e. $\left.\lambda_{0}=\max \operatorname{Re} \sigma\left(A_{0}\right)\right)$ then $\lambda(\varepsilon)$ is convex. Moreover, it is strictly convex unless $A_{1}$ is a scalar matrix and $\lambda(\varepsilon)$ is linear.

Received by the editors November 24, 1989.

1980 Mathematics Subject Classification (1985 Revision). Primary 47A55, 47A10; Secondary 47B55, 47B50, 15A18.

This research, partially supported by SIZ SRH, was finished while the author was visiting the University of Calgary. 
See [2, 3] and also [4] where an extension to infinite dimensional spaces is given.

In this note we shall prove two related results:

(c) If $X$ is a Krein space, $A(\varepsilon)$ self-adjoint for $\varepsilon$ real and has separated spectrum (for the definitions see $\S 1$ ) and $\lambda_{0}$ is the maximum of the negative type spectrum $\sigma_{-}\left(A_{0}\right)$ (minimum of the positive type spectrum $\sigma_{+}\left(A_{0}\right)$, resp.) then $\lambda(\varepsilon)$ is convex (concave, resp.). Moreover, it is strictly convex (strictly concave, resp.) unless $A_{0} x_{0}=\lambda_{0} x_{0}$ implies $A_{1} x_{0}=\lambda_{1} x_{0}$, hence $\lambda(\varepsilon)$ is linear.

(d) If $X=\mathbf{C}^{n}, A_{0}$ is an irreducible essentially nonnegative matrix, $A_{1}$ and $B$ are diagonal matrices and $\lambda_{0}$ is the left distinguished eigenvalue $\lambda_{-}$ (the right distinguished eigenvalue $\lambda_{+}$) of the generalized eigenvalue problem

$$
A_{0} x=\lambda B x
$$

and if $\lambda(\varepsilon)$ is the eigenvalue of the generalized eigenvalue problem

$$
\left(A_{0}+\varepsilon A_{1}\right) x=\lambda B x
$$

such that $\lim _{\varepsilon \rightarrow 0} \lambda(\varepsilon)=\lambda_{0}$, then $\lambda(\varepsilon)$ is convex (concave, resp.). Moreover, it is strictly convex (strictly concave, resp.) unless $A_{1}$ and $B$ are scalar matrices in which case $\lambda(\varepsilon)$ is linear.

For the definition of the distinguished eigenvalues of the generalized eigenvalue problem, see $\S 2$.

\section{EXTREMAL EIGENVALUES OF A FAMILY OF SELF-ADJOINT OPERATORS WITH SEPARATED SPECTRUM IN A KREIN SPACE}

Let $X$ be a Krein space with inner product [ | ] and intrinsic norm \|\| (see [1] for these and subsequent notions). Let $A_{0}$ be a self-adjoint operator in $X$ such that there exist a real number $\gamma$ and a positive constant $C$ such that

$$
\left[\left(A_{0}-\gamma I\right) x \mid x\right] \geq C\|x\|^{2} \quad\left(x \in D\left(A_{0}\right)\right)
$$

holds. Then the spectrum $\sigma\left(A_{0}\right)$ is separated:

$$
\sigma\left(A_{0}\right)=\sigma_{-}\left(A_{0}\right) \cup \sigma_{+}\left(A_{0}\right),
$$

$\sigma_{-}\left(A_{0}\right)=(-\infty, \gamma) \cap \sigma\left(A_{0}\right), \sigma_{+}\left(A_{0}\right)=(\gamma, \infty) \cap \sigma\left(A_{0}\right)$, and $\sigma_{-}\left(A_{0}\right) \quad\left(\sigma_{+}\left(A_{0}\right)\right.$, resp.) is the set of spectral points of negative (positive, resp.) type. Denote

$$
\lambda_{-}=\max \sigma_{-}\left(A_{0}\right), \quad \lambda_{+}=\min \sigma_{+}\left(A_{0}\right) .
$$

Proposition 1. Let $A_{0}$ be a self-adjoint operator in $X$ such that (1) holds and let $A_{1}$ be a bounded self-adjoint operator. Assume that $\lambda_{0}=\lambda_{-}\left(\lambda_{0}=\lambda_{+}\right.$, resp. $)$is an isolated simple eigenvalue of $A_{0}$. Then there exist an interval $J=\left(-\varepsilon_{0}, \varepsilon_{0}\right)$ and an analytic function $\lambda_{-}(\varepsilon) \quad\left(\lambda_{+}(\varepsilon)\right.$, resp.) on $J$ such that $\lambda_{-}(0)=\lambda_{0}$ $\left(\lambda_{+}(0)=\lambda_{0}\right.$, resp. $)$ and $\lambda_{-}(\varepsilon)\left(\lambda_{+}(\varepsilon)\right.$, resp. $)$ is an isolated simple eigenvalue of $A(\varepsilon):=A_{0}+\varepsilon A_{1}(\varepsilon \in J)$ which is the maximum of $\sigma_{+}(A(\varepsilon)$ ) (minimum of 
$\sigma_{-}(A(\varepsilon))$, resp. $) ; \lambda_{-}(\varepsilon)$ is convex $\left(\lambda_{+}(\varepsilon)\right.$ is concave, resp. $)$. It is strictly convex (strictly concave, resp.) unless $A_{0} x_{0}=\lambda_{0} x_{0}$ implies $A_{1} x_{0}=\lambda_{1} x_{0}$, hence $\lambda_{-}(\varepsilon)$ $\left(\lambda_{+}(\varepsilon)\right.$, resp. $)$ is linear.

Proof. First note that $A(\varepsilon)$ also satisfies (1) (with a different $C$ ) if $|\varepsilon|$ is small enough and that the analytic eigenvalue $\lambda(\varepsilon)$ such that $\lambda(0)=\lambda_{0}$ is a simple eigenvalue of negative type which is the maximum of $\sigma_{-}(A(\varepsilon))$. Hence $\lambda(\varepsilon)=$ $\lambda_{-}(\varepsilon)$. Let $x(\varepsilon)=x_{0}+\varepsilon x_{1}+\varepsilon^{2} x_{2}+\cdots$ be the analytic eigenvector function corresponding to $\lambda(\varepsilon)$ such that $\left[x_{0} \mid x_{0}\right]=-1$. Let $\lambda(\varepsilon)=\lambda_{0}+\varepsilon \lambda_{1}+\varepsilon^{2} \lambda_{2}+\cdots$. The eigenvalue equation

$$
A(\varepsilon) x(\varepsilon)=\lambda(\varepsilon) x(\varepsilon)
$$

yields the well-known equalities

$$
\begin{aligned}
A_{0} x_{0} & =\lambda_{0} x_{0}, \\
A_{1} x_{0}+A_{0} x_{1} & =\lambda_{1} x_{0}+\lambda_{0} x_{1}, \\
A_{1} x_{1}+A_{0} x_{2} & =\lambda_{2} x_{0}+\lambda_{1} x_{1}+\lambda_{0} x_{2},
\end{aligned}
$$

implying

$$
\begin{aligned}
\lambda_{1} & =-\left[A_{1} x_{0} \mid x_{0}\right], \\
\left(A_{0}-\lambda_{0}\right) x_{1} & =\left(\lambda_{1}-A_{1}\right) x_{0}, \\
\lambda_{2} & =-\left[\left(A_{1}-\lambda_{1}\right) x_{1} \mid x_{0}\right] .
\end{aligned}
$$

Using the spectral function $E$ of the definitizable operator $A_{0}$ (see [1]) it follows from the last two of these equalities that

$$
\lambda_{2}=\int_{-\infty}^{\infty} \frac{1}{\lambda_{0}-t} d\left[E(t) y_{0} \mid y_{0}\right]
$$

with $y_{0}=\left(A_{1}-\lambda_{1}\right) x_{0}$. Since $\left[E(\cdot) y_{0} \mid y_{0}\right]$ is monotone increasing on $\sigma_{+}\left(A_{0}\right)$, monotone decreasing on $\sigma_{-}\left(A_{0}\right)$, it follows that the integral is nonpositive; it is equal to zero if and only if $y_{0}=0$. This proves all the statements about $\lambda_{+}(\varepsilon)$; the other case is treated analogously.

The boundedness assumption on $A_{1}$ can be relaxed: we used only the analyticity of $\lambda(\varepsilon)$ and the fact that $A(\varepsilon)$ also satisfies (1).

Example. The Klein-Gordon operator with a small potential. Let $V \in L^{\infty}\left(R^{n}\right)$ and $\lim _{|x| \rightarrow \infty} V(x)=0$ and let $X=L^{2}\left(R^{n}\right) \times L^{2}\left(R^{n}\right)$. Endowed with the inner product

$$
[x \mid y]=\left(x_{1} \mid y_{2}\right)_{L^{2}}+\left(x_{2} \mid y_{1}\right)_{L^{2}} \quad\left(x=\left(x_{1}, x_{2}\right), y=\left(y_{1}, y_{2}\right)\right),
$$

$X$ is a Krein space. Let $m^{2}>0$. Define $H=\left(-\Delta+m^{2}\right)^{1 / 2}$ as the positive square root of the positive definite operator $-\Delta+m^{2}$ in $L^{2}\left(\mathbf{R}^{n}\right)$ (defined on $\left.H^{2}\left(\mathbf{R}^{n}\right)\right)$. Then $\mathscr{D}(H)=H^{1}\left(\mathbf{R}^{n}\right)$. Define the operator $A_{0}$ on $\mathscr{D}\left(A_{0}\right)=H^{1}\left(\mathbf{R}^{n}\right) \times H^{1}\left(\mathbf{R}^{n}\right)$ :

$$
A_{0}\left(x_{1}, x_{2}\right)=\left(V x_{1}+H x_{2}, H x_{1}+V x_{2}\right) \quad\left(x=\left(x_{1}, x_{2}\right) \in \mathscr{D}\left(A_{0}\right)\right) .
$$


This operator is easily seen to be self-adjoint in $X$. Let $A_{1}$ be the bounded operator:

$$
A_{1}\left(x_{1}, x_{2}\right)=\left(V x_{1}, x_{2}\right) \quad\left(\left(x_{1}, x_{2}\right) \in X\right) .
$$

It is not hard to verify that $A_{0}$ satisfies the assumptions of Proposition 1 if $\|V\|_{L^{\infty}}$ is sufficiently small ( $\|V\|_{L^{\infty}}<m$ is sufficient). The operator $A_{0}$ is connected with the Klein-Gordon equation and the form $[x \mid x]$ with the charge of the particle represented by the equation (cf. [6] for the equation and [5] for the eigenvalues of the Klein-Gordon operators). The family $A(\varepsilon)=A_{0}+$ $\varepsilon A_{1}$ describes the effect of changing potential. The eigenvalue $\lambda_{+}(\varepsilon)$ at the bottom of the positive spectrum is the analogue of the ground state energy of the Schrödinger operator. By Proposition 1 it follows that $\lambda_{+}(\varepsilon)$ is concave (the ground state energy is concave by the result in (a) in the introduction).

\section{Distinguished eigenvalues of a generalized eigenvalue PRoblem}

Let $X=\mathbf{C}^{n}$ and let $A_{0}$ be an irreducible essentially nonnegative matrix, $A_{1}$ and $B$ real diagonal matrices. We consider the eigenvalue problem

$$
\left(A_{0}+\varepsilon A_{1}\right) x=\lambda B x \text {. }
$$

Let $\nu(\lambda)$ be the principal eigenvalue of the irreducible essentially nonnegative matrix $A_{0}-\lambda B$ (see (b) in the introduction); it is a convex function. If additionally

$$
\nu(0)<0
$$

and $B$ is indefinite, then there are exactly two real values $\lambda$ such that $\nu(\lambda)=0$; denote them $\lambda_{-}, \lambda_{+}$so that $\lambda_{-}<0<\lambda_{+}$. We call these two eigenvalues the left and right distinguished eigenvalue of the generalized eigenvalue problem $A x=\lambda B x$; this notion was used by Kato in [4]. These two eigenvalues have the following properties: $\lambda_{-}\left(\lambda_{+}\right.$, resp. $)$is geometrically simple, it is the largest negative (smallest positive, resp.) eigenvalue, its eigenvector is positive (up to a constant); moreover,

$$
\nu^{\prime}\left(\lambda_{-}\right)<0 \quad\left(\nu^{\prime}\left(\lambda_{+}\right)>0, \text { resp. }\right) .
$$

We are interested in the perturbation of the distinguished eigenvalues. Let $\mu(\lambda, \varepsilon)$ be the principal eigenvalue of $A_{0}+\varepsilon A_{1}-\lambda B$. Then $\nu(\lambda)=\mu(\lambda, 0)$ and for sufficiently small (real) $\varepsilon$ the two distinguished eigenvalues $\lambda_{ \pm}(\varepsilon)$ of the generalized eigenvalue problem (2) exist and are analytic in $\varepsilon$.

Proposition 2. Let $A_{0}$ be an irreducible essentially nonnegative matrix with a negative principal eigenvalue. Let $A_{1}$ and $B$ be real diagonal matrices and let $\lambda_{ \pm}(\varepsilon)$ be the distinguished eigenvalues of the perturbed eigenvalue problem (2). Then $\lambda_{-}(\varepsilon)$ is convex and $\lambda_{+}(\varepsilon)$ is concave. Moreover $\lambda_{-}(\varepsilon)$ is strictly convex and $\lambda_{+}(\varepsilon)$ strictly concave unless $A_{1}$ and $B$ are scalar matrices in which case both $\lambda_{-}^{+}(\varepsilon)$ and $\lambda_{+}(\varepsilon)$ are linear.

Proof. We shall prove the proposition only for $\lambda_{-(}(\varepsilon)=: \lambda(\varepsilon)$. Denote $\lambda(0)=$ : $\lambda_{0}$. Since $\mu$ is analytic in $\lambda, \varepsilon$ and $\lambda(\varepsilon)$ is defined implicitly by $\mu(\lambda, \varepsilon)=0$ 
and since $(\partial \mu / \partial \lambda)\left(\lambda_{0}, 0\right)=\nu^{\prime}\left(\lambda_{0}\right)<0$ it is evident that $\lambda(\varepsilon)$ is analytic. Next we prove that $\mu(\cdot, \cdot)$ is a convex function; this follows from the fact that the function $\lambda \mapsto \mu(\lambda, 0)$ is convex as well as all the functions $\varepsilon \mapsto \mu\left(\lambda_{0}+\alpha \varepsilon, \varepsilon\right)$ for $\alpha \in \mathbf{R}$. This follows from the result quoted in (b) in the introduction; all these functions are strictly convex unless there exist real constants $\alpha, \beta$, and $\gamma$, such that $|\alpha|+|\beta|+|\gamma|>0$ and $\alpha A_{1}+\beta B=\gamma I$ and moreover, one of the above functions is linear. A simple calculation shows that in this case $\nu(\lambda)$ is also linear; hence $A_{1}$ and consequently $B$ are scalar matrices and $\mu(\lambda, \varepsilon)$ is linear, also.

Next note the formula for $\lambda^{\prime \prime}(\varepsilon)$ :

$$
\lambda^{\prime \prime}(\varepsilon)=-\frac{1}{[(\partial \mu / \partial \lambda)(\lambda(\varepsilon), \varepsilon)]^{3}}\left(D^{2} \mu(\lambda(\varepsilon), \varepsilon) D \mu(\lambda(\varepsilon), \varepsilon) \mid D \mu(\lambda(\varepsilon), \varepsilon)\right) .
$$

We know that $(\partial \mu / \partial \lambda)(\lambda(\varepsilon), \varepsilon)$ is negative and $D^{2} \mu(\lambda(\varepsilon), \varepsilon)$ is positive definite near $\left(\lambda_{0}, 0\right)$ (unless $\mu(\lambda, \varepsilon)$ is linear). This proves that either $\lambda^{\prime \prime}(\varepsilon)=0$ or $\lambda^{\prime \prime}(\varepsilon)>0$ for all $\varepsilon$ with $|\varepsilon|$ sufficiently small.

Remarks. 1. The conclusions of Proposition 2 also hold in the situation described in [4, Remark 6.4] where $X=L^{p}(\Omega)$ or $C_{0}(\Omega), A_{0}$ is a second order elliptic partial differential operator in a domain $\Omega$, defined using the Dirichlet boundary conditions, $A_{1}$ and $B$ are multiplication operators.

2 . The eigenvalues $\lambda_{ \pm}(\varepsilon)$ have convexity properties analogous to those described in $\S 1$ and in case (a) in the introduction.

\section{ACKNOWLEDGMENT}

The author is grateful to P. Hess, Zürich, and H. Langer, Dresden, for a helpful discussion.

\section{REFERENCES}

1. J. Bognar, Indefinite inner product spaces, Springer-Verlag, Berlin, Heidelberg, and New York, 1974.

2. J. E. Cohen, Convexity of the dominant eigenvalue of an essentially nonnegative matrix, Proc. Amer. Math. Soc. 81 (1981), 657-658.

3. T. Kato, $A$ short introduction to the perturbation theory of linear operators, Springer-Verlag. Berline. Heidelberg, and New York, 1982.

4. Superconvexity of the spectral radius and convexity of the spectral hound and the type, Math. Z. 180 (1982), 265-273.

5. B. Najman, Eigenvalues of the Klein-Gordon equation, Proc. Edinburgh Math. Soc. 26 (1983), 181-190.

6. K. Veselić, A spectral theory for the Klein-Gordon equation with an external electrostatic potential, Nuclear Phys. A. 147 (1970), 215-224.

Department of Mathematics, University of Calgary, Calgary, Alberta. T2N IN4, CANADA 